\title{
Characterizations of probabilistic and cardinal-probabilistic interaction indices
}

\author{
Katsushige Fujimoto \\ Fukushima University \\ 1 Kanayagawa \\ Fukushima 960-1296, Japan \\ fujimotoe \\ econ.fukushima-u.ac.jp
}

\author{
Ivan Kojadinovic \\ Polytech Nantes \\ LINA CNRS FRE 2729 \\ 44306 Nantes, France \\ Ivan. Kojadinovic@ \\ polytech.univ-nantes.fr
}

\author{
Jean-Luc Marichal \\ University of Luxembourg \\ 162A, avenue de la Faëncerie \\ L-1511 Luxembourg \\ G.D. Luxembourg \\ marichal@cu.lu
}

\begin{abstract}
In the framework of cooperative game theory and multicriteria decision making, the concept of interaction index, which can be regarded as an extension of that of value, has been recently proposed to measure the interaction phenomena among players or criteria. Axiomatizations of two classes of interaction indices, namely probabilistic interaction indices and cardinalprobabilistic interaction indices, generalizing probabilistic values and semivalues, respectively, are first proposed. Three existing instances of cardinal-probabilistic interaction indices encountered thus far in the literature are also axiomatized.
\end{abstract}

Key words : Cooperative games, multicriteria decision making, interaction among players/criteria, values and interaction indices.

\section{Introduction}

The study of the notion of interaction among players is relatively recent in the framework of cooperative game theory. The first attempt is probably due to Owen [14, §5] for superadditive games. More recent developments are due to Murofushi and Soneda [12], Roubens [15], Grabisch [5], and Marichal and Roubens [11] and led successively to the concepts of Shapley interaction index, Banzhaf interaction in- dex, and chaining interaction index. First axiomatic characterizations of the Shapley interaction index and the Banzhaf interaction index have been recently proposed by Grabisch and Roubens [7].

The concept of interaction index, which can be seen as an extension of the notion of value $[1,3,16,17]$, is fundamental for it enables to measure the interaction phenomena ${ }^{1}$ modelled by a game on a set of players.

In this paper, we propose axiomatizations of two families of interaction indices introduced by Grabisch and Roubens [8], namely the broad class of probabilistic interaction indices and the narrower subclass of cardinalprobabilistic interaction indices obtained by additionally imposing the symmetry axiom. Probabilistic interaction indices can be seen as extensions of probabilistic values studied by Weber [17]. Cardinal-probabilistic interaction indices are generalizations of semivalues, which were axiomatized by Dubey et al. [3]. We also separately characterize the Shapley, Banzhaf, and chaining interaction indices, which are instances of cardinal-probabilistic interaction indices.

Besides classical axioms such as linearity and additivity, the axioms involved in the characterizations we present can be regarded as natural generalizations of those used in the axiomatizations of values. Two of the most important axioms in the proposed char-

\footnotetext{
${ }^{1}$ The expression "interaction phenomena" refers to complementarity effects or redundancy effects among players of coalitions resulting from the non additivity of the underlying game.
} 
acterizations of probabilistic and cardinalprobabilistic interaction indices are the $k$ monotonicity axiom, generalizing the monotonicity axiom [17, §4] (called positivity in [9, $\S 4]$ ), and the dummy partnership axiom, which extends the dummy player axiom through the concept of partnership (see e.g. [9]). The notion of partnership is also at the root of some of the axioms additionally imposed to characterize the Shapley, Banzhaf, and chaining interaction indices.

This paper is organized as follows. In the next section we recall some basic definitions and results we will use in this paper. Section 3 is devoted to the concept of interaction index. An intuitive approach is adopted to present this notion and the axiomatizations by Grabisch and Roubens [7] are recalled. In the last section we present our characterization results ${ }^{2}$. Probabilistic and cardinal-probabilistic interaction indices are first axiomatized. Then, the Shapley, Banzhaf, and chaining interaction indices are characterized by imposing additional axioms.

In order to avoid a heavy notation, we adopt that used in [7]. Thus, we will often omit braces for singletons, e.g., by writing $v(i), U \backslash i$ instead of $v(\{i\}), U \backslash\{i\}$. Similarly, for pairs, we will write $i j$ instead of $\{i, j\}$. Furthermore, cardinalities of subsets $S, T, \ldots$, will be denoted by the corresponding lower case letters $s, t, \ldots$

\section{Preliminary definitions}

We consider an infinite set $U$, the universe of players. As usual, a game on $U$ is a set function $v: 2^{U} \rightarrow \mathbb{R}$ such that $v(\varnothing)=0$, which assigns to each coalition $S \subseteq U$ its worth $v(S)$.

We now recall some concepts and results we will use throughout.

\subsection{Carriers}

A set $N \subseteq U$ is said to be a carrier (or support) of a game $v$ when, for all $S \subseteq U$, $v(S)=v(N \cap S)$. Thus, a game $v$ with carrier

\footnotetext{
${ }^{2}$ The proofs of the theorems presented in the sequel are available at www.math.byu.edu/ marichal $/$.
}

$N \subseteq U$ is completely defined by the knowledge of the coefficients $\{v(S)\}_{S \subseteq N}$ and the players outside $N$ have no influence on the game since they do not contribute to any coalition.

In this paper, we restrict our attention to finite games, that is, games that possess finite carriers. We denote by $\mathcal{G}$ the set of finite games on $U$ and by $\mathcal{G}^{N}$ the set of games with finite carrier $N \subseteq U$.

\subsection{Discrete derivatives}

Given a game $v \in \mathcal{G}^{N}$ and finite coalitions $S, T \subseteq U$, we denote by $\Delta_{S} v(T)$ the $S$ derivative of $v$ at $T$, which is recursively defined by

$$
\begin{aligned}
\Delta_{i} v(T) & :=v(T \cup i)-v(T \backslash i), \forall i \in U, \text { and } \\
\Delta_{S} v(T) & :=\Delta_{i}\left[\Delta_{S \backslash i} v(T)\right], \forall i \in S,
\end{aligned}
$$

with convention $\Delta_{\varnothing} v(T):=v(T)$; see $[4, \S 1]$ and $[6, \S 2]$.

We can easily prove by induction on $s$ that, $\forall T \subseteq U \backslash S$,

$$
\Delta_{S} v(T)=\sum_{L \subseteq S}(-1)^{s-l} v(T \cup L) .
$$

It is also easy to show that $\Delta_{S} v(T)=0, \forall S \nsubseteq$ $N, \forall T \subseteq U \backslash S$.

\section{$2.3 \quad k$-monotonicity}

Let $k \geq 2$ be an integer. A game $v \in \mathcal{G}^{N}$ is said to be $k$-monotone (see e.g. [2, §2]) if, for any $k$ coalitions $A_{1}, A_{2}, \ldots, A_{k} \subseteq U$, we have

$$
v\left(\bigcup_{i=1}^{k} A_{i}\right) \geq \sum_{\substack{J \subseteq\{1, \ldots, k\} \\ J \neq \varnothing}}(-1)^{j+1} v\left(\bigcap_{i \in J} A_{i}\right) .
$$

It is easy to verify $[2, \S 2]$ that $k$-monotonicity, with any $k \geq 2$, implies $l$-monotonicity for all $l \in\{2, \ldots, k\}$. By extension, 1-monotonicity (which does not correspond to $k=1$ in Eq. (1)) is defined as standard monotonicity : $v(S) \leq v(T)$ whenever $S \subseteq T \subseteq U$.

Clearly, a game $v \in \mathcal{G}$ is 1-monotone if and only if $\Delta_{i} v(T) \geq 0$ for all $i \in U$ and all $T \subseteq U \backslash i$. For $k$-monotonicity $(k \geq 2)$ we have the following result : 
Proposition 2.1 Let $k \geq 2$. A game $v \in \mathcal{G}$ is $k$-monotone if and only if, for all $S \subseteq U$ such that $2 \leq s \leq k$ and all $T \subseteq U \backslash S$, we have $\Delta_{S} v(T) \geq 0$.

\subsection{Unanimity games}

Consider the set $\mathcal{G}^{N}$ of games. The unanimity game for $T \subseteq N, T \neq \varnothing$, is defined as the game $u_{T} \in \mathcal{G}^{N}$ such that, for all $S \subseteq N$, $u_{T}(S):=1$ if and only if $S \supseteq T$ and 0 otherwise.

\subsection{Permuted games}

Following Shapley [16, §2], given a game $v \in$ $\mathcal{G}^{N}$ and a permutation $\pi$ on $U$ (i.e., a one-toone mapping from $U$ onto itself), we denote by $\pi v$ the game defined by $\pi v[\pi(S)]:=v(S)$, $\forall S \subseteq N$, where $\pi(S):=\{\pi(i) \mid i \in S\}$.

\subsection{Restricted and reduced games}

Given a game $v \in \mathcal{G}^{N}$ and a coalition $A \subseteq N$, the restriction of $v$ to $A[7]$ is a game of $\mathcal{G}^{A}$ defined by $v^{A}(S):=v(S), \forall S \subseteq A$.

Given a coalition $B \subseteq N \backslash A$, the restriction of $v$ to $A$ in the presence of $B$ [7] is a game of $\mathcal{G}^{A}$ defined by $v_{\cup B}^{A}(S):=v(S \cup B)-v(B)$, $\forall S \subseteq A$.

Given a game $v \in \mathcal{G}^{N}$ and a coalition $T \subseteq$ $N, T \neq \varnothing$, the reduced game with respect to $T[7,13]$, denoted $v_{[T]}$, is a game of $\mathcal{G}^{(N \backslash T) \cup[T]}$ where $[T]$ indicates a single hypothetical player, which is the representative (or macro player) of the players in $T$. It is defined by

$$
\begin{aligned}
& v_{[T]}(S):=v(S), \\
& v_{[T]}(S \cup[T]):=v(S \cup T), \quad \forall S \subseteq N \backslash T .
\end{aligned}
$$

\subsection{Dummy coalition, null coalition, partnership, and dummy partnership}

A coalition $S \subseteq U$ is said to be dummy in a game $v \in \mathcal{G}^{N}$ if $v(T \cup S)=v(T)+v(S)$ for all $T \subseteq U \backslash S$. In other words, the marginal contribution of a dummy coalition $S$ to any coalition $T$ not containing elements of $S$ is simply its worth $v(S)$.
A coalition $S \subseteq U$ in a game $v \in \mathcal{G}^{N}$ is said to be null if it is a dummy coalition in $v$ such that $v(S)=0$.

A dummy (resp. null) player is a dummy (resp. null) one-membered coalition.

A coalition $P \subseteq U, P \neq \varnothing$, is said to be a partnership $[9, \S 4]$ in a game $v \in \mathcal{G}^{N}$ if, for all $S \varsubsetneqq P, v(S \cup T)=v(T)$ for all $T \subseteq U \backslash P$. In other words, as long as all the members of a partnership $P$ are not all in coalition, the presence of some of them only leaves unchanged the worth of any coalition not containing elements of $P$. Thus, a partnership behaves like a single hypothetical player, that is, the game $v$ and its reduced version $v_{[P]}$ can be considered as equivalent.

Now, a dummy partnership is simply a partnership $P \subseteq U$ that is dummy. Thus, a dummy partnership can be regarded as a single hypothetical dummy player.

\section{The concept of interaction index}

\subsection{Intuitive presentation}

As noticed by Grabisch and Roubens [7], the fact that in general, for a player $i \in N$ in a game $v \in \mathcal{G}^{N}$, the value of $i$ in $v$ (see e.g. $[1,3,16,17])$ is not equal to the coefficient $v(i)$ shows that players in $N$ have some interest in forming coalitions. For instance, consider another player $j \in N$ and assume that $v(i)$ and $v(j)$ are small whereas $v(i j)$ is large. Then, $i$ and $j$ have clearly a strong interest in joining together. Conversely, it may happen that $v(i)$ and $v(j)$ are large whereas $v(i j)$ is small, in which case $i$ and $j$ have no interest in joining together.

In order to intuitively approach the concept of interaction, consider two players $i$ and $j$ such that $v(i j)>v(i)+v(j)$. Clearly, the above inequality seems to model a positive interaction or complementary effect between $i$ and $j$. Similarly, the inequality $v(i j)<v(i)+v(j)$ suggests considering that $i$ and $j$ interact in a negative or redundant way. Finally, if $v(i j)=$ $v(i)+v(j)$, it seems natural to consider that players $i$ and $j$ do not interact, i.e., that they have independent roles in the game.

A coefficient measuring the interaction be- 
tween $i$ and $j$ should therefore depend on the difference $v(i j)-[v(i)+v(j)]$. However, as discussed by Grabisch and Roubens [7], the intuitive concept of interaction requires a more elaborate definition. Clearly, one should not only compare $v(i j)$ and $v(i)+v(j)$ but also see what happens when $i, j$, and $i j$ join coalitions. In other words, an index of interaction between $i$ and $j$ in the game $v \in \mathcal{G}^{N}$ should take into account all the coefficients of the form $v(T \cup i), v(T \cup j)$, and $v(T \cup i j)$, with $T \subseteq N \backslash i j$.

Owen $[14, \S 5]$ defined an interaction index between two players $i j \subseteq N$ in a game $v \in \mathcal{G}^{N}$ by

$$
I(v, i j):=\sum_{T \subseteq N \backslash i j} \frac{(n-t-2) ! t !}{(n-1) !} \Delta_{i j} v(T) .
$$

Notice that, for a coalition $T$ not containing $i$ and $j$, the expression

$\Delta_{i j} v(T)=v(T \cup i j)-v(T \cup i)-v(T \cup j)+v(T)$

can be regarded as the difference between the marginal contributions $\Delta_{j} v(T \cup i)=v(T \cup$ $i j)-v(T \cup i)$ and $\Delta_{j} v(T)=v(T \cup j)-v(T)$. Following Grabisch et al. [6, §2], we shall call this expression the marginal interaction between $i$ and $j$ in the presence of $T$. Indeed, it seems natural to consider that if $\Delta_{j} v(T \cup i)>$ $\Delta_{j} v(T)$ (resp. $<$ ) then $i$ and $j$ interact positively (resp. negatively) in the presence of $T$ since the presence of player $i$ increases (resp. decreases) the marginal contribution of $j$ to coalition $T$.

The interaction index proposed by Owen, which was actually rediscovered twenty years later by Murofushi and Soneda [12], can thus be regarded as a weighted average of the marginal interactions between $i$ and $j$ in the presence of $T$, all coalitions $T$ not containing $i$ and $j$ being considered.

Grabisch [5] recently extended the above interaction index to coalitions containing more than two players. The Shapley interaction index [5] of a coalition $S \subseteq N$ in a game $v \in \mathcal{G}^{N}$ is defined by

$$
I_{S h}(v, S):=\sum_{T \subseteq N \backslash S} \frac{(n-t-s) ! t !}{(n-s+1) !} \Delta_{S} v(T) .
$$

This index is an extension of the Shapley value in the sense that $I_{S h}(v, i)$ and the Shapley value of $i$ in $v$ coincide for all $i \in U$ and all $v \in \mathcal{G}$. For $S \subseteq N, s \geq 2$, it can be interpreted as a weighted average of $\Delta_{S} v(T)$, which can be regarded as the marginal interaction among players in $S$ in the presence of $T$. More details can on the interpretation of interaction indices can be found in $[6,10]$.

Two similar indices are due to Roubens [15] and Marichal and Roubens [11] and are known as the Banzhaf interaction index and the chaining interaction index ${ }^{3}$, respectively. The former extends the Banzhaf value, while the latter (also) extends the Shapley value. The Banzhaf interaction index [15] and the chaining interaction index [11] of a coalition $S \subseteq N$ in a game $v \in \mathcal{G}^{N}$ are respectively defined by

$$
\begin{aligned}
& I_{B}(v, S):=\sum_{T \subseteq N \backslash S} \frac{1}{2^{n-s}} \Delta_{S} v(T), \\
& I_{c h}(v, S):= \\
& \sum_{T \subseteq N \backslash S} s \frac{(n-s-t) !(s+t-1) !}{n !} \Delta_{S} v(T) .
\end{aligned}
$$

If $S \nsubseteq N$, we naturally set $I_{S h}(v, S):=0$, $I_{B}(v, S):=0$, and $I_{c h}(v, S):=0$.

\subsection{Probabilistic and cardinal probabilistic interaction indices}

By analogy with the work of Dubey et al. [3] and Weber [17] on values, Grabisch and Roubens [8] defined the class of probabilistic interaction indices and the subclass of cardinal-probabilistic interaction indices.

A probabilistic interaction index of a coalition $S \subseteq N$ in a game $v \in \mathcal{G}^{N}$ is of the form

$$
I_{p}(v, S):=\sum_{T \subseteq N \backslash S} p_{T}^{S}(N) \Delta_{S} v(T),
$$

where, for any $S \subseteq N$, the family of coefficients $\left\{p_{T}^{S}(N)\right\}_{T \subseteq N \backslash S}$ forms a probability distribution on $2^{N \backslash \bar{S}}$. Here again, if $S \nsubseteq N$, we naturally set $I_{P}(v, S):=0$.

\footnotetext{
${ }^{3}$ Notice that the chaining interaction index was initially defined in terms of maximal chains of the ordered set $\left(2^{N}, \subseteq\right)$.
} 
A cardinal-probabilistic interaction index is a probabilistic interaction index such that, additionally, for any $S \subseteq N$, the coefficients $p_{T}^{S}(N)(T \subseteq N \backslash S)$ depend only on the cardinal of the coalitions $S, T$, and $N$, i.e., for any $s \in\{0, \ldots, n\}$, there exists a family of nonnegative real numbers $\left\{p_{t}^{s}(n)\right\}_{t=0, \ldots, n-s}$ fulfilling

$$
\sum_{t=0}^{n-s}\left(\begin{array}{c}
n-s \\
t
\end{array}\right) p_{t}^{s}(n)=1
$$

such that, for any $S \subseteq N$ and any $T \subseteq N \backslash S$, we have $p_{T}^{S}(N)=p_{t}^{S}(n)$.

The Shapley, Banzhaf, and chaining interaction indices defined above are clearly cardinalprobabilistic interaction indices.

\subsection{Existing characterizations}

Setting $\mathcal{U}:=2^{U} \backslash\{\varnothing\}$, an interaction index can be regarded as a function $I: \mathcal{G} \times \mathcal{U} \rightarrow \mathbb{R}$ such that, for any $v \in \mathcal{G}$ and any $i \in U, I(v, i)$ is the value of player $i$ in the game $v$, and for any $S \subseteq U$ such that $s \geq 2, I(v, S)$ is a measure of the (simultaneous) interaction among players in $S$ in the game $v$.

Grabisch and Roubens recently proposed an axiomatic characterization of the Shapley and the Banzhaf interaction indices [7, §3]. We present their results hereafter, with the only difference that here we force the second argument of $I$ to be nonempty. The following axioms have been considered by Grabisch and Roubens :

- Linearity axiom $(L): I$ is a linear function with respect to its first argument.

- Dummy player axiom (D): If $i \in U$ is a dummy player in a game $v \in \mathcal{G}$, then

(i) $I(v, i)=v(i)$,

(ii) for all $S \subseteq U \backslash i, S \neq \varnothing$, we have $I(v, S \cup i)=0$.

- Symmetry axiom $(S)$ : For any permutation $\pi$ on $U$, and any $v \in \mathcal{G}$, we have $I(v, S)=I(\pi v, \pi(S))$ for all $S \subseteq U, S \neq$ $\varnothing$.
- Recursive axiom (R) : For all finite $N \subseteq$ $U, n \geq 2$, for all $v \in \mathcal{G}^{N}$, we have, $\forall S \subseteq$ $N, s \geq 2, \forall j \in S$,

$$
I(v, S)=I\left(v_{\cup j}^{N \backslash j}, S \backslash j\right)-I\left(v^{N \backslash j}, S \backslash j\right) .
$$

- Efficiency (E) : For all finite $N \subseteq U, n \geq$ 1 , and all $v \in \mathcal{G}^{N}$, we have

$$
\sum_{i \in N} I(v, i)=v(N) \text {. }
$$

- 2-efficiency (2-E) : For all finite $N \subseteq U$, $n \geq 2$, and all $v \in \mathcal{G}^{N}$, we have

$$
I(v, i)+I(v, j)=I\left(v_{[i j]},[i j]\right), \quad \forall i j \subseteq N .
$$

The following theorem was shown by Grabisch and Roubens in $[7, \S 3]$ :

Theorem 3.1 Let I be a function from $\mathcal{G} \times \mathcal{U}$ to $\mathbb{R}$.

(i) I satisfies axiom ( $L$ ) if and only if, for any finite $N \subseteq U, n \geq 1$, and any $S \subseteq N$, $s \geq 1$, there exists a family of real constants $\left\{\alpha_{T}^{S}(N)\right\}_{T \subseteq N}$ such that, for any $v \in \mathcal{G}^{N}$, we have

$$
I(v, S)=\sum_{T \subseteq N} \alpha_{T}^{S}(N) v(T) .
$$

(ii) I satisfies axioms (L) and (D), if and only if, for any finite $N \subseteq U, n \geq 1$, and any $S \subseteq N, s \geq 1$, there exists a family of constants $\left\{p_{T}^{S}(N)\right\}_{T \subseteq N \backslash S}$ such that, for any $v \in \mathcal{G}^{N}$, we have

$$
I(v, S)=\sum_{T \subseteq N \backslash S} p_{T}^{S}(N) \Delta_{S} v(T),
$$

and for any $S \nsubseteq N, s \geq 1$, and any $v \in$ $\mathcal{G}^{N}$, we have $I(v, S)=0$.

(iii) I satisfies axioms (L), (D), and (S), if and only if, for any finite $N \subseteq U, n \geq 1$, and any $S \subseteq N, s \geq 1$, there exists a family of constants $\left\{p_{t}^{s}(n)\right\}_{t=0, \ldots, n-s}$ such that, for any $v \in \mathcal{G}^{N}$, we have

$$
I(v, S)=\sum_{T \subseteq N \backslash S} p_{t}^{s}(n) \Delta_{S} v(T),
$$

and for any $S \nsubseteq N, s \geq 1$, and any $v \in$ $\mathcal{G}^{N}$, we have $I(v, S)=0$. 
(iv) I satisfies axioms $(L),(D),(S),(R)$, and (E) if and only if $I=I_{S h}$.

(v) I satisfies axioms (L), (D), (S), (R), and (2-E) if and only if $I=I_{B}$.

Parts (iv) and (v) of Theorem 3.1 thus provide axiomatic characterizations of the Shapley and Banzhaf interaction indices respectively. ${ }^{4}$

\section{New axiomatic characterizations}

\subsection{Probabilistic and cardinal probabilistic interaction indices}

We shall now axiomatize the class of probabilistic interaction indices and that of cardinal-probabilistic interaction indices. The following axioms are first considered :

- Additivity axiom (A) : $I$ is an additive function with respect to its first argument.

- Monotonicity axiom (M) : For any monotone game $v \in \mathcal{G}$, we have $I(v, i) \geq 0$ for all $i \in U$.

- $k$-monotonicity axiom $\left(M^{k}\right)$ : For any $k \geq$ 2 and any $k$-monotone game $v \in \mathcal{G}$, we have $I(v, S) \geq 0$ for all coalition $S \subseteq U$ such that $2 \leq s \leq k$.

Axiom (A) indicates that interaction indices should be decomposable additively whenever games are decomposable additively. Axiom $(\mathrm{M})$, used by Weber in $[17, \S 4]$ to characterize probabilistic values, concerns only the value part of $I$ and states that, since in a monotone game the marginal contributions of a player are necessarily positive, its value should be positive. Axiom $\left(\mathrm{M}^{k}\right)$ can be seen as a generalization of axiom (M) and concerns the interaction part of $I$. As discussed in [10], in

\footnotetext{
${ }^{4}$ It is noteworthy that, since axiom $(R)$ determines uniquely $I(v, S), s \geq 2$, from the values $I(v, i), i \in N$, the axioms $(L),(D)$, and $(S)$ are somewhat redundant in parts (iv) and (v) and are needed only for values $I(v, i), i \in N$.
}

a $k$-monotone game $(k \geq 2)$, it seems sensible to consider that there are necessarily complementarity effects among players of coalitions containing between 2 and $k$ players. Axiom $\left(\mathrm{M}^{k}\right)$ then simply states that these effects should be represented as positive interactions.

We also consider the following fundamental axiom :

- Dummy partnership axiom (DP) : For any $v \in \mathcal{G}$, if $P \neq \varnothing$ is a dummy partnership in $v$, then

(i) $I(v, P)=v(P)$,

(ii) for all $S \subseteq U \backslash P, S \neq \varnothing$, we have $I(v, S \cup P)=0$.

Axiom (DP) is a natural generalization of axiom (D). As discussed by Weber [17, §3], the first part of axiom (D) is based on the following intuition : since the marginal contribution of a dummy player to any coalition not containing it is simply its worth, its value should be its worth as well. Similarly, the first part of axiom (DP) states that the interaction index of a dummy partnership $P$ in a game $v$ should be its worth since the marginal interaction among the players in $P$ in the presence of any coalition $T$ not containing elements of $P$ is its worth, that is, $\Delta_{P} v(T)=v(P)$.

The second part of axiom (DP) is a natural extension of the second part of axiom (D) and says that there should be no interaction among players of coalitions containing dummy partnerships.

We now provide axiomatic characterizations of probabilistic and cardinal-probabilistic interaction indices.

Theorem 4.1 A function $I: \mathcal{G} \times \mathcal{U} \rightarrow \mathbb{R}$ satisfies axioms $(A),(M),\left(M^{k}\right)$, and $(D P)$ if and only if, for any finite $N \subseteq U, n \geq 1$, and any $S \subseteq N, s \geq 1$, there exists a family of nonnegative real constants $\left\{p_{T}^{S}(N)\right\}_{T \subseteq N \backslash S}$ satisfying $\sum_{T \subseteq N \backslash S} p_{T}^{S}(N)=1$ such that, for any $v \in \mathcal{G}^{N}$, we have

$$
I(v, S)=\sum_{T \subseteq N \backslash S} p_{T}^{S}(N) \Delta_{S} v(T),
$$


and for any $S \nsubseteq N, s \geq 1$, and any $v \in \mathcal{G}^{N}$, we have $I(v, S)=0$.

Theorem 4.2 A function $I: \mathcal{G} \times \mathcal{U} \rightarrow \mathbb{R}$ satisfies axioms $(A),(M),\left(M^{k}\right),(D P)$, and $(S)$ if and only if, for any finite $N \subseteq U, n \geq 1$, and any $S \subseteq N, s \geq 1$, there exists a family of nonnegative real constants $\left\{p_{t}^{s}(n)\right\}_{t=0, \ldots, n-s}$ satisfying $\sum_{t=0}^{n-s}\left(\begin{array}{c}n-s \\ t\end{array}\right) p_{t}^{s}(n)=1$, such that, for any $v \in \mathcal{G}^{N}$, we have

$$
I(v, S)=\sum_{T \subseteq N \backslash S} p_{t}^{s}(n) \Delta_{S} v(T),
$$

and for any $S \nsubseteq N, s \geq 1$, and any $v \in \mathcal{G}^{N}$, we have $I(v, S)=0$.

We shall now proceed with the characterizations of the Shapley, Banzhaf, and chaining interaction indices which, as mentioned before, are all instances of cardinal-probabilistic interaction indices.

\subsection{Shapley and Banzhaf interaction indices}

The following axiom is first additionally considered :

- Reduced-partnership-consistency axiom $(R P C)$ : If $P$ is a partnership in a game $v \in \mathcal{G}$ then $I(v, P)=I\left(v_{[P]},[P]\right)$.

Recall that a partnership can be considered as behaving as a single hypothetical player. Furthermore, it is easy to verify that the marginal interaction among the players of a partnership $P$ in a game $v \in \mathcal{G}^{N}$ in the presence of a coalition $T \subseteq N \backslash P$ is equal to the marginal contribution of $P$ to coalition $T$, i.e., $\Delta_{P} v(T)=v(T \cup P)-v(T)$. In other words, when we measure the interaction among the players of a partnership, it is as if we were measuring the value of an hypothetical player. Axiom (RPC) then simply states that the interaction among players of a partnership $P$ in a game $v$ should be regarded as the value of the reduced partnership $[P]$ in the corresponding reduced game $v_{[P]}$.

We then have the following interesting result :
Proposition 4.1 A function $I: \mathcal{G} \times \mathcal{U} \rightarrow \mathbb{R}$ that satisfies axioms (L), (D) and (RPC) also satisfies axiom (DP).

We now state axiomatic characterizations of the Shapley and Banzhaf interaction indices.

Theorem 4.3 The Shapley interaction index is the only cardinal-probabilistic interaction index additionally satisfying axioms (E) and $(R P C)$. As a consequence, the Shapley interaction index is the only interaction index satisfying axioms $(A),(M),\left(M^{k}\right),(D$ or $D P)$, $(S),(E)$, and $(R P C)$.

Theorem 4.4 The Banzhaf interaction index is the only cardinal-probabilistic interaction index additionally satisfying axioms (2-E) and (RPC). As a consequence, the Banzhaf interaction index is the only interaction index satisfying axioms $(A),(M),\left(M^{k}\right),(D$ or $D P)$, (S), (2-E), and (RPC).

The following interesting result can be used to obtain additional characterizations of the two interaction indices under consideration.

Proposition 4.2 Under axioms (L), (DP), and $(S)$, axioms $(R)$ and $(R P C)$ are equivalent.

\subsection{Banzhaf and chaining interaction indices}

We consider the following additional axiom :

- Partnership-allocation axiom (PA) : If $P$ is a partnership in $v \in \mathcal{G}$ then

$$
I(v, P) I\left(u_{P}, i\right)=I(v, i), \quad \forall i \in P .
$$

Let $I_{p}$ be a cardinal-probabilistic interaction index, $P$ be a partnership in a game $v \in \mathcal{G}^{N}$, and $i$ be a member of $P$. Then axiom (PA) is based on the following intuitions :

1. It is easy to verify that $I_{p}(v, P)$ is a weighted average of the marginal contributions $v(T \cup P)-v(T)(T \subseteq N \backslash P)$ and that $I_{p}(v, i)$ is a weighted sum of these same marginal contributions. In other 
words, both $I_{p}(v, P)$ and $I_{p}(v, i)$ can be considered as measuring the value in the game $v$ of the hypothetical macro player corresponding to $P$.

2. Let $\alpha_{i}$ be the real number such that $I_{p}(v, P)=\alpha_{i} I_{p}(v, i)$. Notice this equality still holds if $i$ is replaced with any other player $j \in P$, since all players in a partnership play symmetric roles. The coefficient $\alpha_{i}$, which depends only on $P$ and $v$, can then be seen as determining the way $I_{p}(v, P)$ is calculated from the value of any of the players of the partnership, quantity that contains all the "relevant information" as discussed in Point 1.

3. It could then be required that the way the value of $P$ is determined from the value of a player of the partnership does not depend on the underlying game but only on $P$. Coalition $P$ being clearly a (dummy) partnership in the unanimity game $u_{P}$, we immediately obtain that $\alpha_{i} I_{p}\left(u_{P}, i\right)=$ 1 , which justifies axiom (PA).

We now state another characterization of the Banzhaf interaction index and a characterization of the chaining interaction index.

Theorem 4.5 The Banzhaf interaction index is the only cardinal-probabilistic interaction index additionally satisfying axioms (2-E) and (PA). As a consequence, the Banzhaf interaction index is the only interaction index satisfying axioms $(A),(M),\left(M^{k}\right),(D P),(S),(2-E)$, and $(P A)$.

Theorem 4.6 The chaining interaction index is the only cardinal-probabilistic interaction index additionally satisfying axioms (E) and $(P A)$. As a consequence, the chaining interaction index is the only interaction index satisfying axioms $(A),(M),\left(M^{k}\right),(D P),(S)$, $(E)$, and (PA).

\section{Conclusion}

Axiomatic characterizations of the broad class of probabilistic interaction indices and of the narrower subclass of cardinal-probabilistic interaction indices have been proposed. The presented characterizations are based on natural generalizations of the monotonicity and dummy player axioms, namely, the $k$ monotonicity and the dummy partnership axioms. Then, by further imposing classical axioms such as efficiency, 2-efficiency, and additional axioms based on the concept of partnership, we have characterized the Shapley, Banzhaf, and chaining interactions indices, which are the three best-known instances of cardinal-probabilistic interaction indices.

\section{References}

[1] J. Banzhaf. Weighted voting doesn't work: A mathematical analysis. Rutgers Law Review, 19:317-343, 1965.

[2] A. Chateauneuf and J.-Y. Jaffray. Some characterizations of lower probabilities and other monotone capacities through the use of Mbius inversion. Math. Social Sci., 17(3):263-283, 1989.

[3] P. Dubey, A. Neyman, and R. J. Weber. Value theory without efficiency. Math. Oper. Res., 6(1):122-128, 1981.

[4] S. Foldes and P. L. Hammer. Submodularity, supermodularity, higher order monotonicities. Submitted, 2002.

[5] M. Grabisch. $k$-order additive discrete fuzzy measures and their representation. Fuzzy Sets and Systems, 92(2):167-189, 1997.

[6] M. Grabisch, J.-L. Marichal, and M. Roubens. Equivalent representations of set functions. Math. Oper. Res., 25(2):157178,2000 .

[7] M. Grabisch and M. Roubens. An axiomatic approach to the concept of interaction among players in cooperative games. Internat. J. Game Theory, 28(4):547-565, 1999.

[8] M. Grabisch and M. Roubens. Probabilistic interactions among players of a cooperative game. In Beliefs, interactions and preferences in decision making (Mons, 1997), volume 40 of Theory Decis. Lib. Ser. B Math. Statist. Methods, pages 205-216. Kluwer Acad. Publ., Dordrecht, 1999.

[9] E. Kalai and D. Samet. Weighted Shapley values. In The Shapley value, pages 83-99. Cambridge Univ. Press, Cambridge, 1988

[10] I. Kojadinovic. Modeling interaction phenomena using fuzzy measures: on the notions of interation and independence. Fuzzy Sets and Systems, 135(3):317-340, 2002

[11] J.-L. Marichal and M. Roubens. The chaining interaction index among players in cooperative games. In Advances in decision analysis (Mons, 1997), volume 4 of Math. Model. Theory Appl., pages 69-85. Kluwer Acad. Publ., Dordrecht, 1999.

[12] T. Murofushi and S. Soneda. Techniques for reading fuzzy measures (iii): interaction index. In 9th Fuzzy System Symposium, pages 693-696, Saporo, Japan, 1993.

[13] A. S. Nowak. On an axiomatization of the Banzhaf value without the additivity axiom. Internat. J. Game Theory, 26(1):137-141, 1997 .

[14] G. Owen. Multilinear extensions of games. Management Sci., 18:P64-P79, 1971/72

[15] M. Roubens. Interaction between criteria and definition of weights in MCDA problems. In 44th meeting of the european working group "Multicriteria Aid for Decisions", Brussels, Belgium, October 1996.

[16] L. S. Shapley. A value for $n$-person games. In Contributions to the theory of games, vol. 2, Annals of Mathematics Studies, no. 28, pages 307-317. Princeton University Press, Princeton, no. J., 1953

[17] R. J. Weber. Probabilistic values for games. In The Shapley value, pages 101-119. Cambridge Univ. Press, Cambridge, 1988 